\title{
Differential Emission Measure Plasma Diagnostics of a Long-Lived Coronal Hole
}

\author{
Jonas Saqri ${ }^{1}\left(\right.$ D $\cdot$ Astrid M. Veronig $^{1}$ (D) \\ Stephan G. Heinemann ${ }^{1}$ (D) - Stefan J. Hofmeister ${ }^{1}$ (D) \\ Manuela Temmer ${ }^{1}$ (D) Karin Dissauer $^{1}$ (D) Yang Su$^{2}$ (ID
}

Received: 12 August 2019 / Accepted: 8 December 2019 / Published online: 10 January 2020

(C) The Author(s) 2020

\begin{abstract}
We use Solar Dynamics Observatory (SDO)/Atmospheric Imaging Assembly (AIA) data to reconstruct the plasma properties from differential emission measure (DEM) analysis for a previously studied long-lived, low-latitude coronal hole $(\mathrm{CH})$ over its lifetime of ten solar rotations. We initially obtain a non-isothermal DEM distribution with a dominant component centered around $0.9 \mathrm{MK}$ and a secondary smaller component at 1.5-2.0 MK. We find that deconvolving the data with the instrument point spread function (PSF) to account for long-range scattered light reduces the secondary hot component. Using the 2012 Venus transit and a 2013 lunar eclipse to test the efficiency of this deconvolution, significant amounts of residual stray light are found for the occulted areas. Accounting for this stray light in the error budget of the different AIA filters further reduces the secondary hot emission, yielding $\mathrm{CH}$ DEM distributions that are close to isothermal with the main contribution centered around 0.9 MK. Based on these DEMs, we analyze the evolution of the emission measure (EM), density, and averaged temperature during the $\mathrm{CH}$ 's lifetime. We find that once the $\mathrm{CH}$ is clearly observed in EUV images, the bulk of the $\mathrm{CH}$ plasma reveals a quite constant state, i.e. temperature and density reveal no major changes, whereas the total $\mathrm{CH}$ area and the photospheric magnetic fine structure inside the $\mathrm{CH}$ show a distinct evolutionary pattern. These findings suggest that $\mathrm{CH}$ plasma properties are mostly "set" at the $\mathrm{CH}$ formation or/and that all $\mathrm{CHs}$ have similar plasma properties.
\end{abstract}

Keywords Corona $\cdot$ Coronal holes

\section{Introduction}

Coronal holes are regions of reduced plasma density and temperature in the solar corona. Thus they appear darker than the surrounding quiet Sun in extreme-ultraviolet (EUV) and

\footnotetext{
$\bowtie$ A.M. Veronig

astrid.veronig@uni-graz.at

Institute of Physics \& Kanzelhöhe Observatory, University of Graz, Graz, Austria

2 Purple Mountain Observatory, Chinese Academy of Sciences (CAS), Nanjing, China
} 
$\mathrm{X}$-ray images. Magnetically, $\mathrm{CHs}$ are characterized by the prevailing dominant polarity nature of the underlying magnetic field where magnetic-field lines of the dominant polarity are "open" into interplanetary space (Wilcox, 1968; Altschuler, Trotter, and Orrall, 1972; Hundhausen, 1972). Along these open magnetic-field lines, plasma is accelerated, escaping the Sun's gravitational field forming interplanetary high-speed solar-wind streams. The solarwind parameters speed and proton temperature measured at $1 \mathrm{AU}$ are correlated with the CH area (Krieger, Timothy, and Roelof, 1973; Vršnak, Temmer, and Veronig, 2007; Rotter et al., 2012).

These open flux tubes are believed to be rooted in concentrations of magnetic flux, known as magnetic elements (Hassler et al., 1999; Tu et al., 2005). Based on a study of 288 lowlatitude CHs, Hofmeister et al. (2017) found that these magnetic elements are also the main origin of the unbalanced magnetic flux of CHs. The total area covered by long-lived magnetic elements with lifetimes $>4$ days is strongly correlated with the unbalanced magnetic flux of CHs ( $\mathrm{cc}=0.99$ ). These magnetic elements account for $\approx 68 \%$ of the unbalanced "open" magnetic flux of CHs at typical coverages of about $3 \%$ of the total $\mathrm{CH}$ area (Hofmeister et al., 2019; Heinemann et al., 2019).

Heinemann et al. (2018a,b) investigated a long-lived low-latitude CH observed by the Solar Dynamics Observatory (SDO) and Solar Terrestrial Relations Observatory (STEREO) spacecraft over ten solar rotations from February 2012 to October 2012 (see Figure 7). Based on the $\mathrm{CH}$ area, they identified a distinct three-phase evolution characterized by a growing, maximum, and decaying phase. For this $\mathrm{CH}$, a strong correlation between $\mathrm{CH}$ area and the mean magnetic-field strength inside the $\mathrm{CH}$ was found. The signed magnetic-field strength increased over the course of the growing phase and reached its maximum at the time of maximal $\mathrm{CH}$ area followed by a steep drop in field strength marking the beginning of the $\mathrm{CH}$ 's decaying phase. During the maximum phase, a larger fraction of the overall magnetic flux is concentrated in magnetic elements than in the growing and decaying phases, and the number of magnetic elements per area for elements with field strengths $>50 \mathrm{G}$ increases nearly $100 \%$ compared to either the beginning or end of the CH's life. This maximum phase further exhibits the strongest correlation between $\mathrm{CH}$ area and peak speed of the associated high-speed solar-wind stream.

As the magnetic-field configuration is assumed to be responsible both for the coronal heating (e.g. Abramenko, Pevtsov, and Romano, 2006; Klimchuk, 2006) and for the acceleration of the solar-wind plasma (Wang, 2010), the clear trend in the magnetic structure and the distinct correlations with the fast solar-wind characteristics of this $\mathrm{CH}$ identified by Heinemann et al. (2018a,b) make it an ideal candidate for probing the effect of changing magnetic fine structure on $\mathrm{CH}$ plasma. The low-latitude position of the $\mathrm{CH}$ under study minimizes projection effects, thus making it also a suitable candidate for differential emission measure (DEM) analysis. This allows us to deduce the temperature-dependent emission of the optically thin coronal plasma by studying multi-wavelength EUV measurements in combination with elemental abundances and the wavelength dependent instrument response.

In this article we apply DEM diagnostics using the inversion code developed by Hannah and Kontar (2012) using SDO/Atmospheric Imaging Assembly (AIA) data to reconstruct the DEM distribution of a $\mathrm{CH}$ and infer the density and temperature evolution over its lifetime. 
Figure 1 Temperature response functions for the utilized AIA EUV filters generated using CHIANTI 9 and the effective areas provided by aia_get_response.pro.

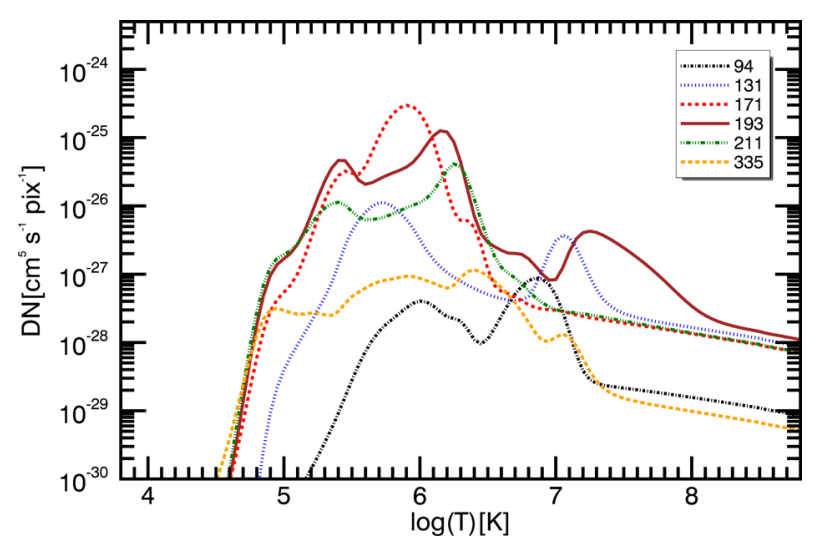

\section{Data and Methods}

\subsection{Data}

We made use of six of the seven EUV channels (94, 131, 171, 193, 211, and $335 \AA$ ) of the AIA instrument (Lemen et al., 2012) onboard the SDO (Pesnell, Thompson, and Chamberlin, 2012) spacecraft, sensitive over a temperature range from $10^{5} \mathrm{~K}$ to $10^{7} \mathrm{~K}$. The $304 \AA$ filter was omitted because it images optically thick plasma making it unsuitable for DEM analysis, which relies on optically thin emission. With the exception of the He II imaging $304 \AA$ channel, AIA's EUV filters primarily observe different Fe ions (with ionization states from Fe VIII to Fe XXIV).

The AIA temperature response functions of the filters utilized are given in Figure 1. The AIA pixel scale is 0.6 arcsec (Lemen et al., 2012). To enhance the signal-to-noise ratio, the data were binned by $8 \times 8$ pixels while conserving flux, resulting in an effective spatial resolution of 4.8 arcsec per pixel. The AIA data were deconvolved with the instrument point spread function (PSF) in order to reduce the instrument stray light and its effect on the reconstructed DEMs (Section 3). The data were further processed using the standard SSWIDL data reduction routine aia_prep.pro.

We then perform the DEM analysis at a cadence of one hour during the \pm 6 -hour time window where the $\mathrm{CH}$ is nearest to the disk center to reduce line-of-sight (LOS) integration effects of the emission of close-by structures. We applied the same $\mathrm{CH}$ boundaries as in Heinemann et al. (2018a,b) which were generated using an intensity-based threshold method applied to the AIA $193 \AA$ images (Rotter et al., 2012; Hofmeister et al., 2017).

\subsection{Differential Emission Measure Analysis}

For optically thin emission from plasma in thermodynamic equilibrium the temperature distribution of the contributing plasma in the LOS is described by the differential emission measure defined as

$$
\operatorname{DEM}(T)=n(T)^{2} \frac{\mathrm{d} h}{\mathrm{~d} T}
$$

where $n$ is the number density dependent on the temperature $T$ along the LOS. The measured intensity $I_{\lambda}(T)$ for a given AIA filter is then related to the DEM via

$$
I_{\lambda}=\int_{T} K_{\lambda}(T) \operatorname{DEM}(T) \mathrm{d} T
$$


where $K_{\lambda}(T)$ denotes the response function of the corresponding filter, which depends on elemental abundances and the temperature of the emitting plasma as well as on the sensor sensitivity (Hannah and Kontar, 2012). We assumed photospheric element abundances, as up to the lower corona CHs show abundances close to photospheric values (Feldman, 1998; Feldman and Widing, 2003). For calculating the instrument response, abundances were taken from the CHIANTI 9 database (Dere et al., 1997, 2019) and the filter response function from aia_get_response.pro in the SolarSoftware (SSWIDL) package. To infer the DEM from AIA data, we applied the regularized inversion technique developed by Hannah and Kontar (2012) to reconstruct the DEM curve for each binned pixel from the six coronal AIA EUV channels. The temperature range considered for DEM analysis was chosen from 0.2 to $5 \mathrm{MK}$. Integration of the DEM over the temperature range yields the total emission measure:

$$
\mathrm{EM}=\int_{T} \operatorname{DEM}(T) \mathrm{d} T
$$

Following Cheng et al. (2012) and Vanninathan et al. (2015), the mean temperature can be estimated by the emission-weighted temperature,

$$
\bar{T}=\frac{\int_{T} \operatorname{DEM}(T) T \mathrm{~d} T}{\operatorname{EM}} .
$$

Assuming a filling factor of unity, the plasma density can then be derived from Equation 1 as

$$
\bar{n}=\sqrt{\frac{\mathrm{EM}}{h}},
$$

with $h$ the column height of emitting plasma along the LOS. As an estimate for the column height of plasma contributing to the measured emission, we use the hydrostatic scale height,

$$
h=\frac{\mathrm{k}_{\mathrm{B}} T}{\mu \mathrm{m}_{\mathrm{H}} g} .
$$

Using $\mu=0.64$ and $\mathrm{m}_{\mathrm{H}}=1.67 \times 10^{-27} \mathrm{~kg}$ for a fully ionized solar plasma and assuming a typical $\mathrm{CH}$ plasma temperature around $0.9 \mathrm{MK}$, one arrives at a typical scale height $h \approx$ $42 \mathrm{Mm}$.

\section{Effect of PSF Deconvolution on Reconstructed DEMs}

Wendeln and Landi (2018) pointed out that any high-temperature component in the $\mathrm{CH}$ plasma distribution inferred by spectroscopic analysis is likely an artifact due to instrument scattered light. In order to reduce the effect of instrument stray light on the recovered DEM, we deconvolved the data with the instrument PSF provided by the aia_calc_psf.pro routine. This is in particular relevant for studying regions of low emission such as CHs. To estimate the effectiveness of the deconvolution in reducing stray light over low-emission regions, we analyze the intensity profiles over areas occulted by the Venus transit on 6 June 2012 and a lunar eclipse on 6 August 2013.

Figure 2 (bottom panels) compares the intensities of data processed solely with aia_prep.pro (level 1.5) with the intensities of the deconvolved data (level 1.6) along white lines indicated in the images in the top panels. Over the areas shaded by Venus (left) or the Moon (right), deconvolution reduces the remaining intensity for most channels, but some 

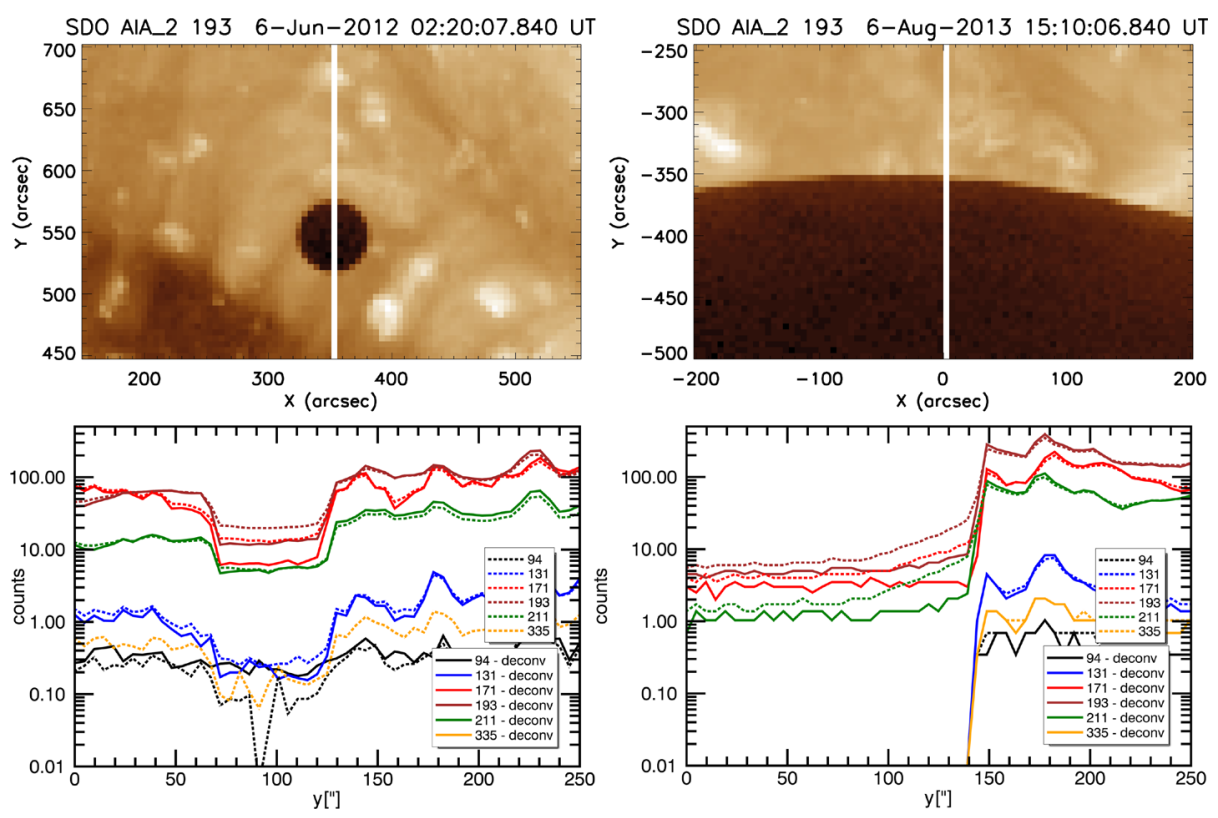

Figure 2 Top: AIA $193 \AA$ images of the Venus transit (left) and lunar eclipse (right). The white vertical lines indicate the regions, from where the intensity profiles shown in the bottom panels were derived. Bottom: Comparison of intensity profiles before and after deconvolution with the PSF for low-emission areas. Left: Intensity profile for the AIA EUV channels along the indicated white line during the 2012 Venus transit. Right: Profile during a lunar eclipse.

DNs still remain owing to long-range scattered light due to the microroughness of the telescope mirrors (private communication, M. Cheung, 2019). The deconvolved data also show better contrast along the edge of the lunar eclipse.

To quantify the significance of the remaining stray light for analyzing $\mathrm{CHs}$, Table 1 shows the remaining DNs after deconvolving with the instrument PSF for typical Venus and lunar pixels compared with the mean DNs of the $\mathrm{CH}$ under study on 6 May 2012 for each AIA channel used. Comparing Venus and $\mathrm{CH}$, for the $131 \AA$ and $171 \AA$ channels, the remaining intensity is small compared to typical CH intensities. For the $193 \AA$ channel the remaining stray light is about $50 \%$ the intensity of typical CH DNs. For the $211 \AA$ and $94 \AA$ channels, the contribution from stray light is about as high as the measured intensity inside the $\mathrm{CH}$. Since the $171 \AA$ and $131 \AA$ filters, which sample cooler plasma inside the CH (Figure 1), are less affected by stray light than the filters sensitive to hotter plasma (Table 1), we conclude that most of the high-temperature emission in the recovered DEM of CHs is likely due to contamination from regions outside the $\mathrm{CH}$.

Figure 3 shows the effect of deconvolving the data with the PSF and accounting for residual stray light on the recovered DEM of CHs. It compares the average DEM for an entire $\mathrm{CH}$ computed with the original, i.e. not-PSF-deconvolved level 1.5 data (red), with the DEM where the PSF was taken into account (blue), and with the DEM where we have additionally increased the presumed errors in each AIA channel due to long-range scattered stray light (green). Thereby, we have used the residual DNs of the Venus transit from Table 1 as an estimate on the long-range scattered light and add these to the error budget of the AIA channels given by the SSWIDL routine aia_bp_estimate_error.pro. We assume this to be a reasonable estimate, as the eclipse happened during the lifetime of the $\mathrm{CH}$ under study, so 
Table 1 Remaining DNs after PSF deconvolution for typical Venus and lunar pixels compared to the mean DNs for a $\mathrm{CH}$ for each used AIA channel.

\begin{tabular}{|c|c|c|c|c|c|c|c|}
\hline Channel & Venus & Lunar & $\mathrm{CH}$ & Channel & Venus & Lunar & $\mathrm{CH}$ \\
\hline $94 \AA ̊$ & 0.2 & 0.0 & 0.3 & $193 \AA$ & 12.4 & 5.1 & 23.5 \\
\hline $131 \AA ̊$ & 0.2 & 0.0 & 1.2 & $211 \AA$ & 5.3 & 1.2 & 6.2 \\
\hline $171 \AA ̊$ & 6.4 & 3.4 & 67.9 & $335 \AA$ & 0.0 & 0.0 & 0.3 \\
\hline
\end{tabular}

Figure 3 Comparison of the DEM averaged over the entire CH (on 15 February 2012) computed from data deconvolved with the instrument PSF (level 1.6, blue) and without deconvolution (level 1.5, black). The high-temperature tail is significantly reduced by the deconvolution as a result of the stray-light correction. Adding the residual DNs determined for the 2012 Venus transit to the uncertainties in the data further reduces the high-temperature emission (green).

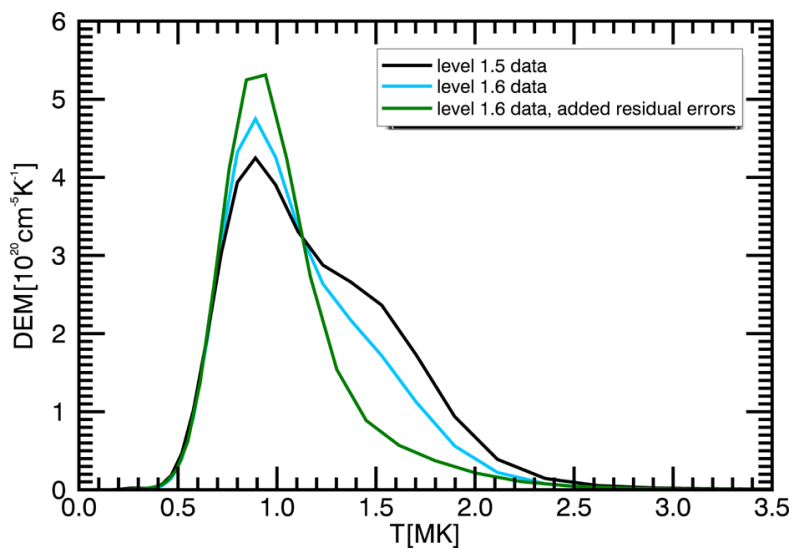

that the EUV emission from the solar disk and thus scattered light are comparable in these data sets. When taking the PSF into account, the high-temperature tail of the $\mathrm{CH}$ plasma is significantly reduced compared to the original data. Considering residual stray light as detailed above, the hot-temperature component even almost vanishes. To compute more accurate DEMs, we therefore account for both PSF and residual stray light.

\section{Results}

\subsection{General Features of the CH DEM Distribution}

Over the ten solar rotations of the CH's lifetime, we derived the DEMs of each $8 \times 8$ binned pixel of the AIA images, when the $\mathrm{CH}$ was close to the central meridian. Figure 4 shows examples of the derived DEM distributions for $\mathrm{CH}$ (top panels) and quiet Sun (QS) pixels (bottom panels). The total EM for $\mathrm{CH}$ regions is about ten times smaller than of the QS with the shape of the DEM also differing. The DEM distribution for $\mathrm{CH}$ regions shows a maximum of the emission at a temperature of around $0.9 \mathrm{MK}$ with a small secondary contribution from emission centered between 1.5-2.0 MK. DEM from QS regions exhibits peak emission between 1.5-2.0 MK with a secondary 0.9-1.1 MK component being sometimes present. Also shown in Figure 4 are the results of fitting the DEMs with the sum of two Gaussian functions centered at those temperatures (solid red and blue lines). The finding that coronal plasma outside CHs also shows emission from a component centered around $\mathrm{CH}$ temperatures is consistent with the reasoning of Landi and Feldman (2008), who concluded that coronal plasma may be a superposition of $\mathrm{CH}, \mathrm{QS}$, and active-region plasma, with CHs missing the hotter components.

We further investigated the dependence of the DEM on the distance from the $\mathrm{CH}$ boundaries. To this aim, we separated the $\mathrm{CH}$ and its immediate surroundings into several segments 

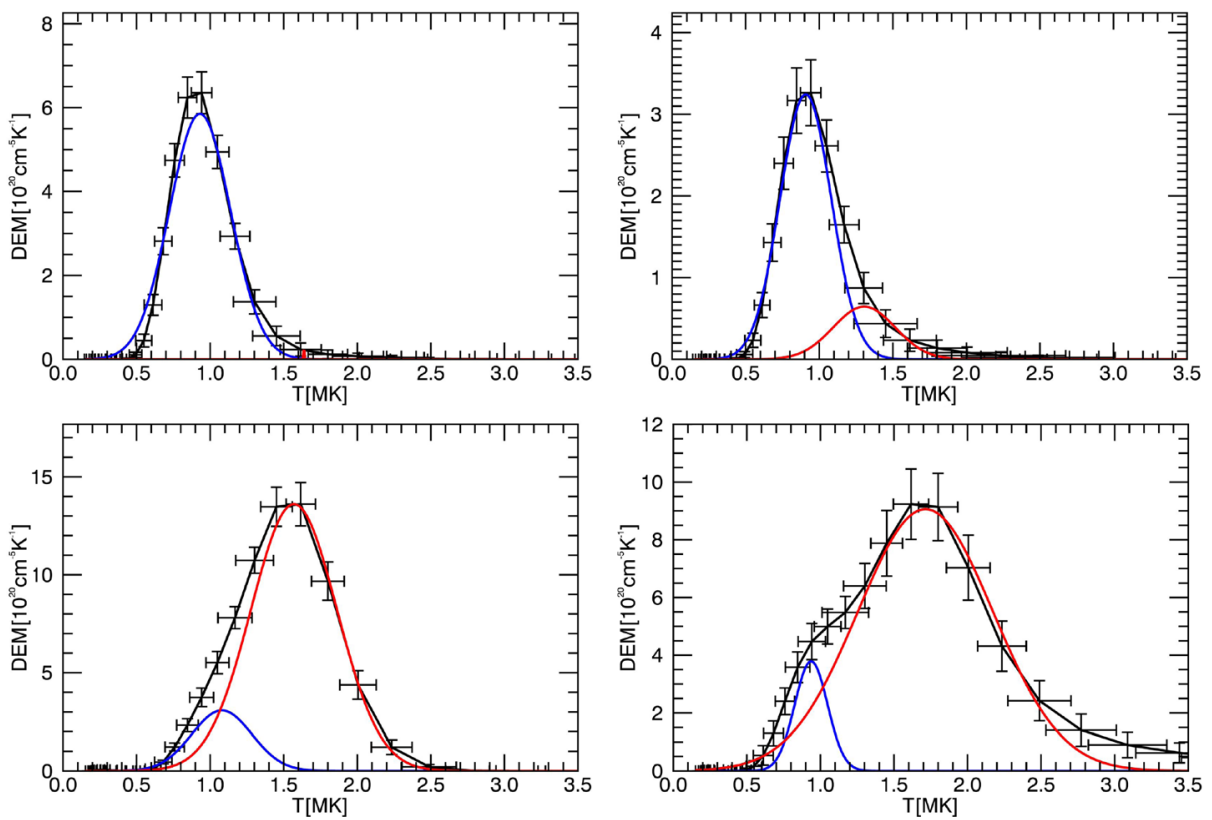

Figure 4 Example of DEM distributions for $\mathrm{CH}$ pixels (top row) and quiet Sun (bottom row) for 30 June 2012 04:00 UT. The red and blue curves are best fits to the DEM using the sum of two Gaussian components. Locations of the chosen pixels are indicated in Figure 7.

outward and inward of the $\mathrm{CH}$ boundary. Inside the $\mathrm{CH}$, each segment has a width of 9.6". For the first region we considered pixels that were just inside the determined $\mathrm{CH}$ boundary up to a distance of 9.6". For the second we used pixels at least 9.6" and at most 19.2" from the boundary, and so on. For the regions outside the $\mathrm{CH}$ boundary shown in black in the upper panel of Figure 5, the widths are 19.2", 19.2", and 38.4". We then took the averages of the DEM for all pixels inside those segments. The resulting averaged DEMs are shown in Figure 5. The segments show a sharp difference between regions inside (red) and outside (blue) the $\mathrm{CH}$. The DEM for segments inside the determined $\mathrm{CH}$ boundary shows a distinct peak at $T \approx 0.9 \mathrm{MK}$ and a small high-temperature tail of constant emission most prominent around $T \approx 1.5-2.0 \mathrm{MK}$. For the segments outside the CH boundary, the total EM is strongly increasing and the contribution from $T \approx 1.5-2.0 \mathrm{MK}$ rises. However, we note that in the segment directly adjacent to the $\mathrm{CH}$ boundary, the $T \approx 0.9 \mathrm{MK}$ peak is still dominant, and only outside $>38.4^{\prime \prime}$ from the $\mathrm{CH}$ boundary the emission peak at $T \approx 1.5-2.0 \mathrm{MK}$ contributes equally. We note that our assumption of photospheric elemental abundances is not fulfilled outside the $\mathrm{CH}$ region, which may affect the derived absolute values and the ratio of cool/hot emission.

The radial profiles for plasma density and DEM-weighted temperature (Equations 4, 5) derived from the averaged DEM curves are also shown in Figure 5. The plasma density decreases towards the interior of the $\mathrm{CH}$ and increases outside the $\mathrm{CH}$ boundary. The temperature inside the $\mathrm{CH}$ is slightly higher towards the $\mathrm{CH}$ center because as the total emission decreases, the constant hot tail becomes relatively more important when calculating the mean temperature from the distribution. As the temperature of the peak does not change and the tail appears to originate mostly from stray light, this increase in plasma temperature towards the $\mathrm{CH}$ center is likely an effect due to limitations of the available data. The temperature 

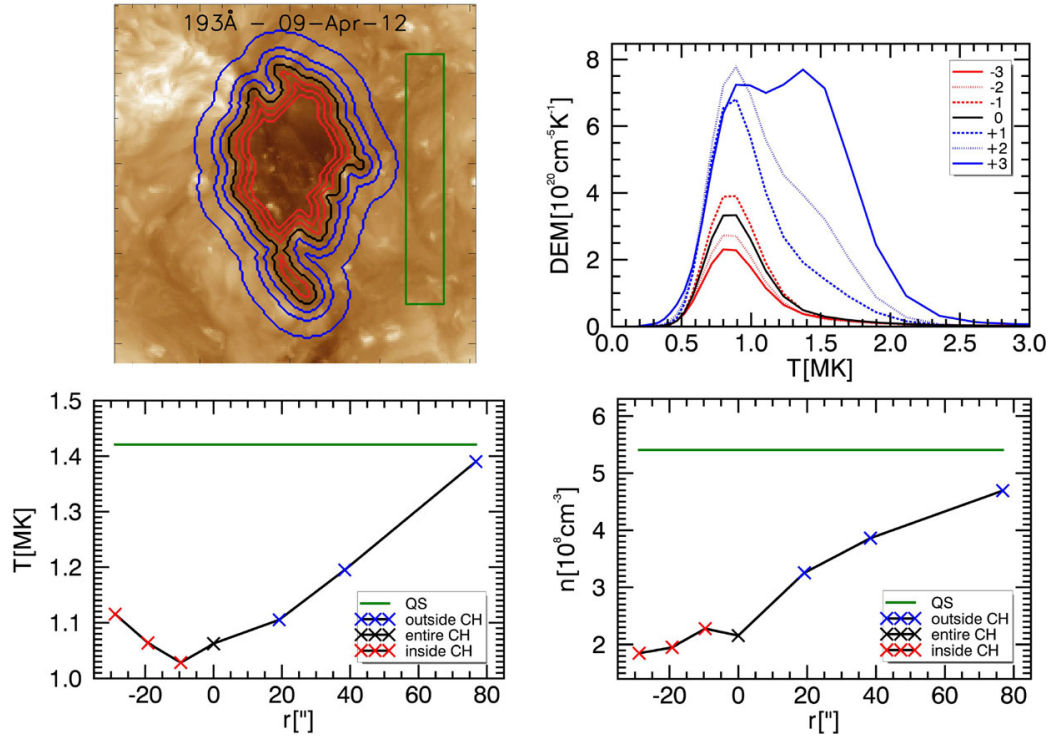

Figure 5 Top row: AIA $193 \AA$ A map (left panel) with contours indicating the regions used for the averaged DEMs shown in the right panel. The map shows a field-of-view of about $750^{\prime \prime} \times 750^{\prime \prime}$. The red curves show inside, blue curves outside regions. The $\mathrm{CH}$ boundary is indicated in black, respectively. The distance between the contours inside the $\mathrm{CH}$ is $9.6^{\prime \prime}$. Outside the $\mathrm{CH}$ boundary, the distances between the contours are $19.2^{\prime \prime}, 19.2^{\prime \prime}$, and $38.4^{\prime \prime}$. Bottom row: Radial profile of DEM-weighted temperature (left) and plasma density (right) within the indicated contours. $r=0^{\prime \prime}$ indicates the $\mathrm{CH}$ boundary. Shown in green are average density and temperature over the QS region indicated by the green rectangle in the top-left panel.

increases again for segments further outside the $\mathrm{CH}$ boundary. For reference, the density and temperature averaged over the QS region (utilizing the same photospheric abundances) indicated by the green rectangle in the top-left panel of Figure 5 are shown in green. As discussed previously, the favored interpretation of the non-vanishing hot $(T \approx 1.5-2.0 \mathrm{MK})$ component inside the $\mathrm{CH}$ seems to be stray light caused by instrumental effects and/or contribution from coronal bright points inside the $\mathrm{CH}$, which show a DEM profile with a main contribution of $T>1.5 \mathrm{MK}$. To reduce the influence of overlying structures and stray light from the surrounding QS, in the further analysis we limited the $\mathrm{CH}$ area used for the DEMs to pixels that are at least three pixels $\left(14.4^{\prime \prime}\right)$ inside any $\mathrm{CH}$ boundary, in addition to deconvolving the data with the instrument PSF and accounting for the residual counts in the error budget as described in Section 2. Using only pixels well inside the $\mathrm{CH}$ has the added benefit of excluding regions where the assumption of photospheric abundances may not be valid. Figure 7 shows these reduced $\mathrm{CH}$ masks overplotted in black alongside the initial $\mathrm{CH}$ masks derived from the image segmentation in white.

Next we investigate the emission measure integrated over different temperature bins to illustrate the described difference in emission between $\mathrm{CH}$ and QS regions. Figure 6 shows the EM maps in $0.2 \mathrm{MK}$ bins over the temperature range $T=0.6$ to $1.8 \mathrm{MK}$ for the $\mathrm{CH}$ on 03 June 2012. In the cooler bins up to 1.2 MK, the $\mathrm{CH}$ does not appear as a distinct region of reduced emission when compared to its surroundings. In contrast, in the EM bins at $T>$ 1.2 $\mathrm{MK}$, the $\mathrm{CH}$ region clearly sticks out as a region of strongly reduced emission compared to the ambient corona. This finding holds for all solar rotations of the CH's lifetime (see Appendix A: Figures 11-19). 


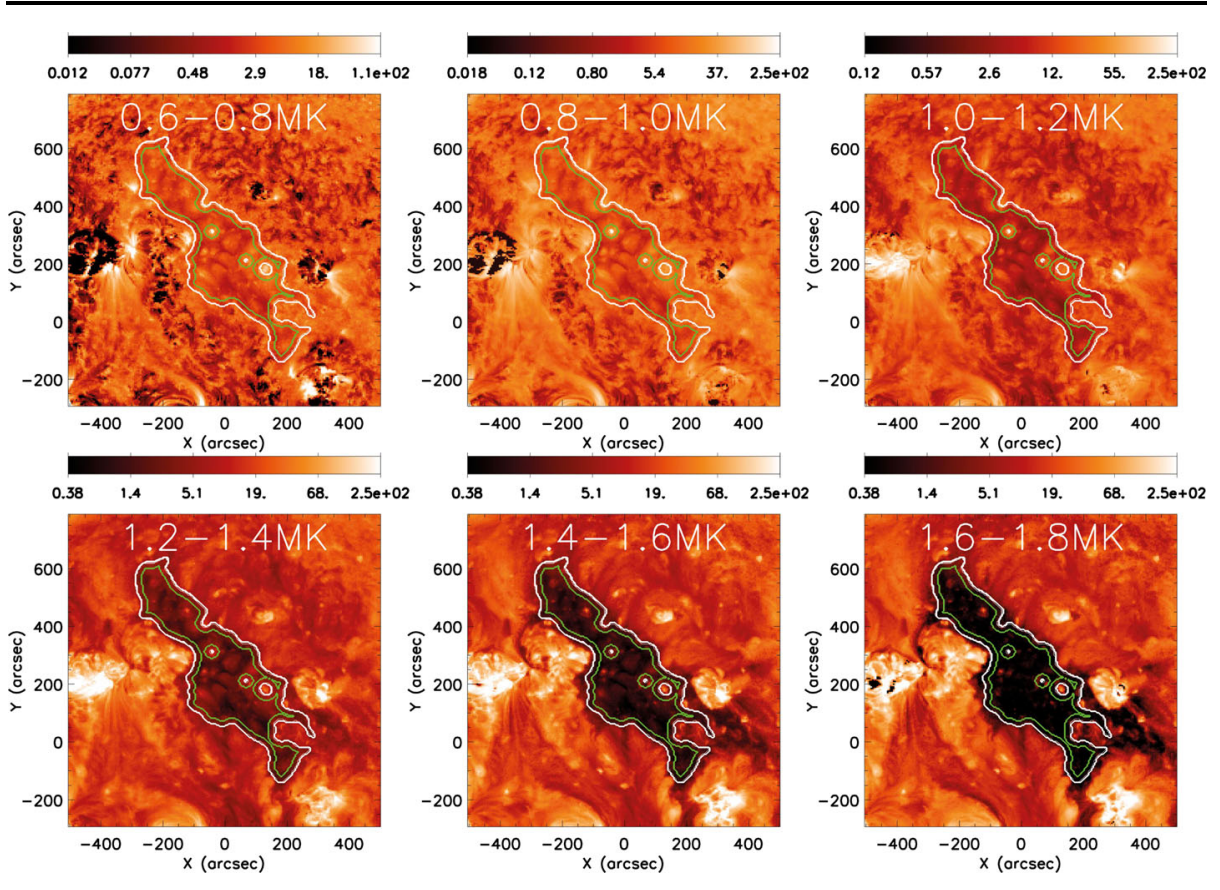

Figure 6 DEM integrated over six different temperature bins in the range 0.6 to $1.8 \mathrm{MK}$ in steps of $0.2 \mathrm{MK}$, yielding the total emission measure (EM) in units $\mathrm{cm}^{-5}$ at those temperature ranges. White contours indicate the initial, green the reduced CH boundaries. Observation from 03 June 2012.

\subsection{Evolution of Plasma Parameters over the CH Lifetime}

Figure 7 shows snapshots of the $\mathrm{CH}$ under study in the AIA $193 \AA$ filter for nine solar rotations from 15 February 2012 to 13 October 2012 illustrating the $\mathrm{CH}$ evolution with a growth of the CH's area followed by a period of maximal area and a decaying phase of diminishing area. $\mathrm{CH}$ boundaries used by Heinemann et al. $(2018 \mathrm{a}, \mathrm{b})$ are indicated in white. Figure 8 shows maps of the mean density and mean ion temperature (Equations 4 and 5) during the $\mathrm{CH}$ evolution. To quantify the changes of the DEM during the $\mathrm{CH}$ 's lifetime, we derive the mean of all of the DEM curves of pixels inside the $\mathrm{CH}$ at a given solar rotation, utilizing the smaller boundaries indicated in black in Figure 7.

Figure 9 shows the derived mean DEMs together with the $1 \sigma$ range of values for the nine solar rotations, shown in Figure 7. It is noted that the DEM in April 2012 is notably reduced. The reason for this steep reduction is unclear but it seems to be a real effect. We tested the DEM of the $\mathrm{CH}$ for different days during this rotation, and all of them showed the same behavior.

Figure 10 shows the evolution of the total EM as well as density and averaged temperature (Equations 3-5) together with the $\mathrm{CH}$ area and fraction of the $\mathrm{CH}$ area covered by magnetic elements $>50 \mathrm{G}$ from Heinemann et al. (2018b) over the $\mathrm{CH}$ lifetime. Error bars indicate standard deviations over different observations within each solar rotation, with data points showing the mean values. EM values are normalized to the maximum of the time series. Comparing the evolution of EM, density and temperature with the evolution of the $\mathrm{CH}$ area and its coverage by magnetic flux concentrations $>50 \mathrm{G}$, there is no clear correlation of the derived evolution of the $\mathrm{CH}$ plasma parameters and the previously 

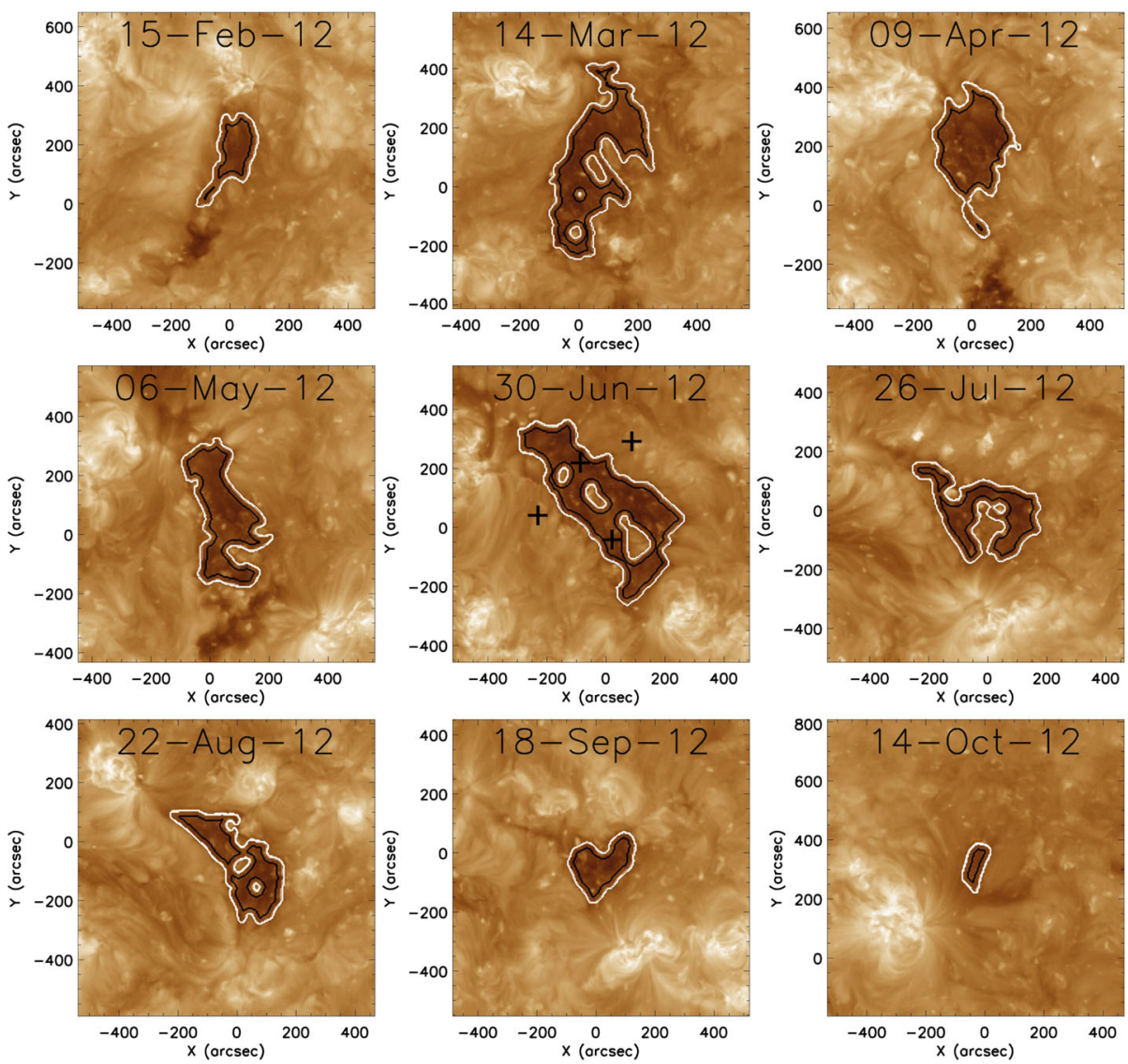

Figure 7 AIA 193 Å filtergrams for nine solar rotations during the lifetime of the $\mathrm{CH}$ under study. The $\mathrm{CH}$ boundaries inferred from the AIA $193 \AA$ Antensity are shown in white. Black lines show regions three pixels inside from the indicated $\mathrm{CH}$ boundary. Pixels for which we show the DEM in Figure 4 are indicated by black crosses.

described phases of growing, maximum, and decaying area identified by Heinemann et al. (2018a,b).

\section{Discussion and Conclusion}

Using the DEM code developed by Hannah and Kontar (2012), we reconstructed mean plasma temperature and density for a long-lived, low-latitude $\mathrm{CH}$ over its lifetime of ten solar rotations from the six coronal AIA EUV filters. We initially recovered a nonisothermal DEM distribution for $\mathrm{CH}$ regions (Figure 4) with the main peak centered around $T=0.9 \mathrm{MK}$ and a secondary smaller component in the range 1.5-2.0 MK. A similar finding was reported in previous studies such as Hahn, Landi, and Savin (2011) who performed DEM analysis of a polar CH using Hinode/Extreme-Ultraviolet Imaging Spectrometer (EIS) data and attributed the hot contribution from 1.5 to $2 \mathrm{MK}$ to structures along the LOS, contamination from streamers or closed loops of higher temperature inside the $\mathrm{CH}$ itself. Wendeln and Landi (2018) studied Hinode/EIS observations of an equatorial $\mathrm{CH}$ 

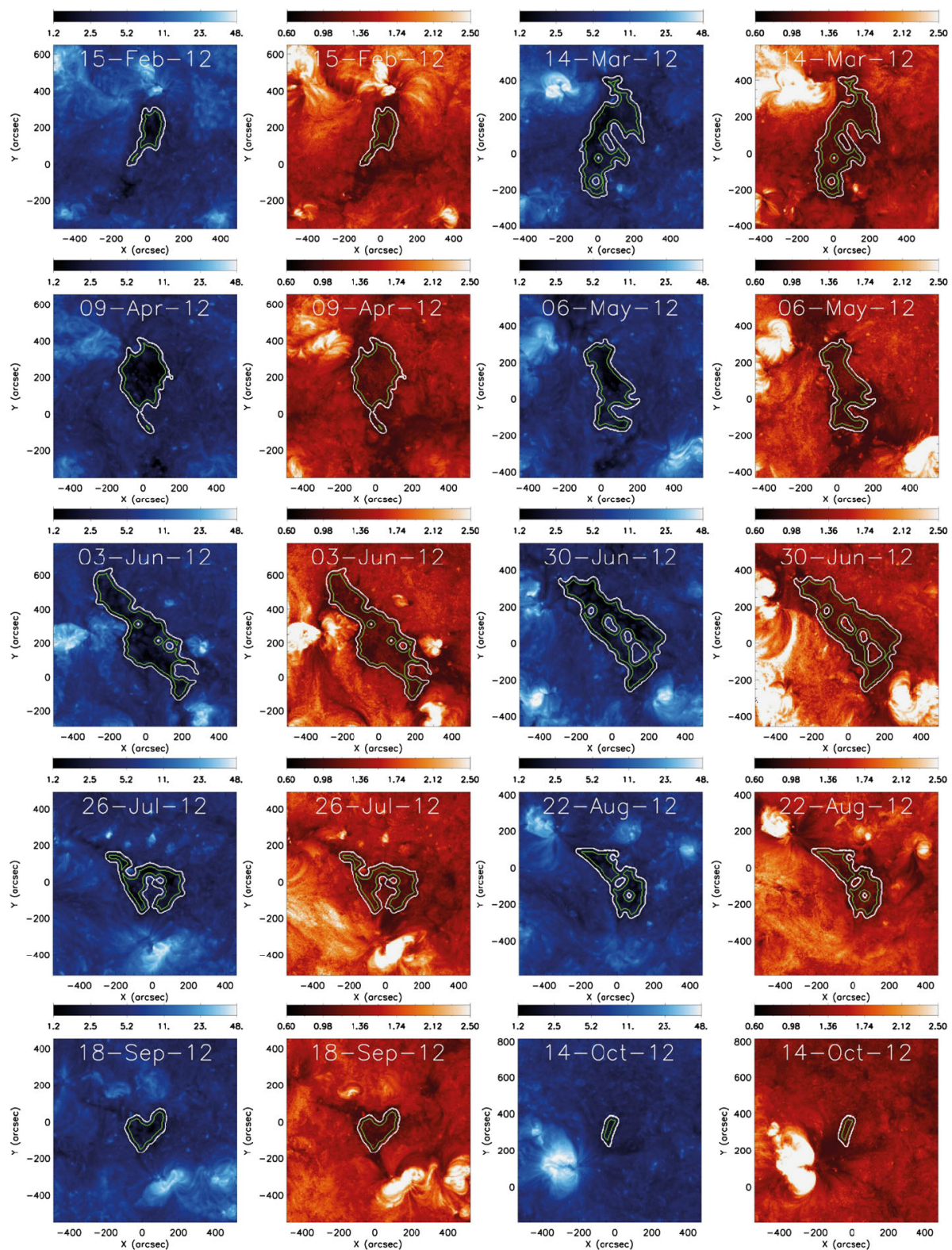

Figure 8 Maps of the mean plasma density [ $\mathrm{cm}^{-3}$ ] (Equation 5) in blue and mean temperatures [MK] (Equation 4) in red together with the initial (white) and reduced (green) $\mathrm{CH}$ boundaries for all ten solar rotations where the $\mathrm{CH}$ was observed.

and concluded that any emission from hot plasma in CHs may be the result of a previously underestimated contribution from instrumental stray light. A study of the off-disk thermal structure for a polar $\mathrm{CH}$ by Landi (2008) using spectra from the Solar and Heliospheric Observatory (SOHO)/Solar Ultraviolet Measurements of Emitted Radiation (SUMER) instrument recovered a near isothermal off-disk $\mathrm{CH}$ plasma with a height-dependent temperature 

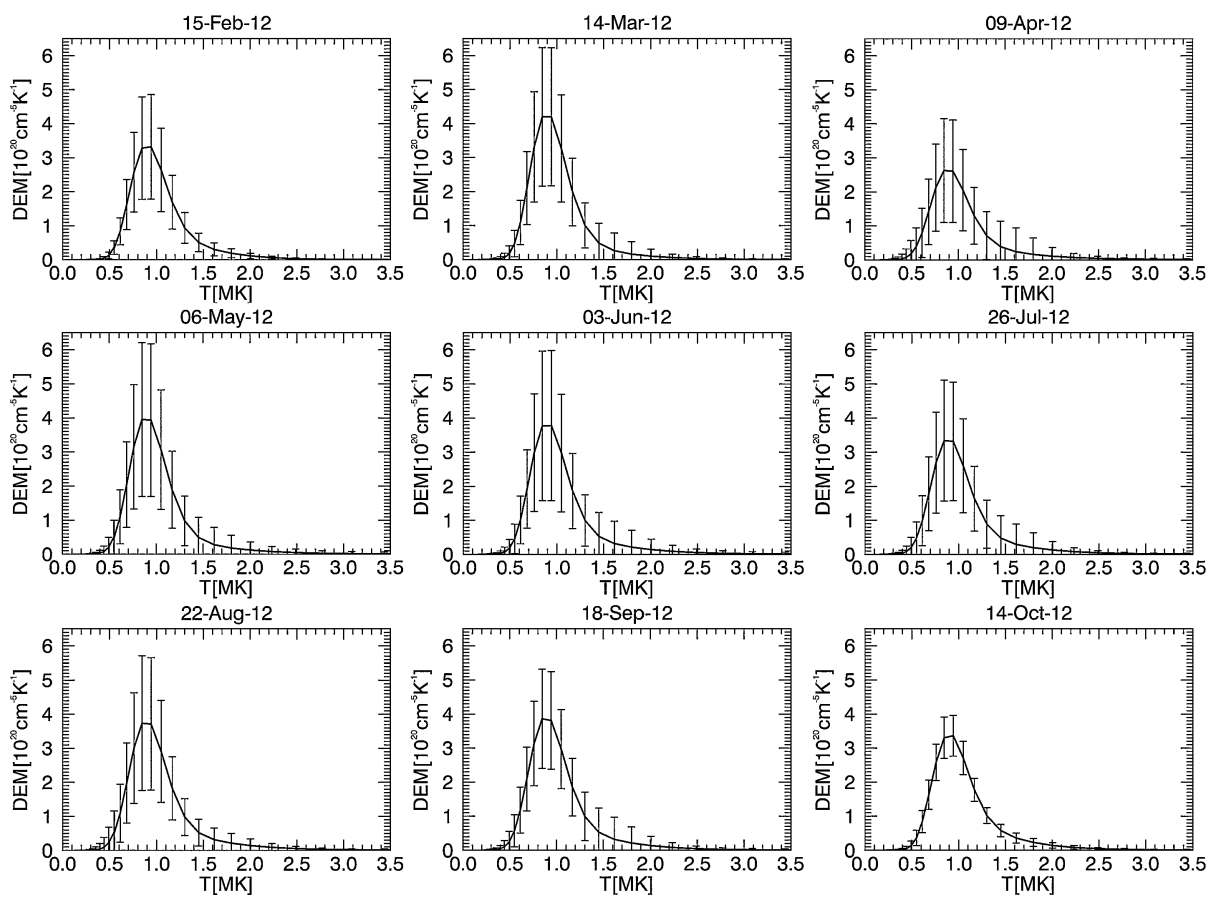

Figure 9 DEM curves averaged over all pixels inside the CH. Note that we used only pixels $14.4^{\prime \prime}$ away from the initial $\mathrm{CH}$ boundary. The evolution over nine solar rotations during the $\mathrm{CH}$ lifetime is shown. Vertical bars indicate the $1 \sigma$ range.

Figure 10 Top: Evolution of the $\mathrm{CH}$ area (black) and fraction of the $\mathrm{CH}$ area covered by magnetic elements > $50 \mathrm{G}$ (red) from Heinemann et al. (2018a,b). Second and third from top: Density and temperature evolution (Equations 4, 5). Fourth panel: Total EM (Equation 3). Error bars show standard deviations for each solar rotation.

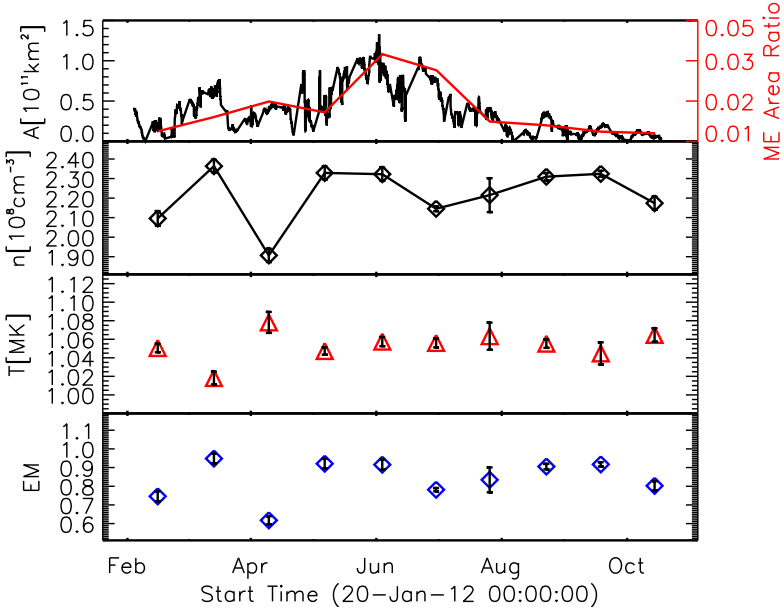

$5.85<\log (T)<6.04 \mathrm{~K}$, further supporting the assumption of a non-physical origin of the hot tail of the recovered CH DEMs.

We showed that deconvolving the data with the instrument PSF reduces the hot tail in the recovered DEM distribution of CHs (Figure 3). Using AIA observations of the 2012 Venus transit and a lunar eclipse, we verified the assumption that the hot tail is not actually emitted 
from the $\mathrm{CH}$. We found that, for hot AIA channels, even after PSF deconvolution significant DNs remain for pixels in regions where the Sun is occulted (Figure 2, Table 1). We therefore used the residual counts obtained by analyzing the radial profile of the Venus transit as an additional contribution to the errors in the AIA measurements, thereby further reducing the hot tail in the derived CH DEM profiles (Figure 3).

EM maps clearly show the $\mathrm{CH}$ as a region of reduced intensity for temperatures $>1.2 \mathrm{MK}$, whereas for emission from cooler temperatures no such effect is noticeable (Figure 6, Appendix A). The radial profile of the density and temperature shows a steep decrease in the regions adjacent to the $\mathrm{CH}$ boundary, with relatively flat profiles inside the $\mathrm{CH}$. While the density decreases away from the boundary, the temperature slightly increases towards the $\mathrm{CH}$ center due to a small, constant, high-temperature emission (Figure 5). As the temperature of the DEM maximum remains constant, we interpret this as the result of instrumental effects that lead to the described constant higher-temperature tail.

We do not find a distinct trend linking $\mathrm{CH}$ area and EM/density or plasma temperature that would correspond to the reported three-phase evolution of the long-lived $\mathrm{CH}$ under study. But we find that once the $\mathrm{CH}$ is clearly observed as a region of reduced emission in the EUV images, it already has a significantly reduced density and temperature, which do not distinctly change over the CH's lifetime. Since the magnetic properties follow the same three-phase evolution as the $\mathrm{CH}$ area (Heinemann et al., 2018b), this indicates that the mean density and temperature evolution of the $\mathrm{CH}$ are not strongly governed by the changing magnetic properties and fine structure of the $\mathrm{CH}$. While the magnetic field is believed to be responsible for heating coronal plasma (Abramenko, Pevtsov, and Romano, 2006), the bulk of the $\mathrm{CH}$ plasma appears to not be significantly altered by the changes in the photospheric magnetic fine structures of the dominant coronal-hole polarity. Our finding that the plasma properties in the $\mathrm{CH}$ do not evolve with the area and magnetic-field strength suggests that the $\mathrm{CH}$ plasma properties are mostly "set" at the $\mathrm{CH}$ formation and/or that all $\mathrm{CHs}$ have similar plasma properties. To verify these hypotheses, a statistical study covering a large set of well observed CHs is necessary.

Acknowledgments Open access funding provided by University of Graz. SDO image data courtesy of NASA/SDO and the AIA science team. A.M. Veronig, S.G. Heinemann, M. Temmer, and K. Dissauer acknowledge the financial support by the Austrian Space Applications Programme of the Austrian Research Promotion Agency FFG (ASAP-13 859729, SWAMI and ASAP-14 865972, SSCME). The authors thank Mark Cheung (LMSAL) for the insightful discussion on the AIA instrument and PSF.

Disclosure of Potential Conflicts of Interest The authors declare that they have no conflicts of interest.

Publisher's Note Springer Nature remains neutral with regard to jurisdictional claims in published maps and institutional affiliations.

Open Access This article is licensed under a Creative Commons Attribution 4.0 International License, which permits use, sharing, adaptation, distribution and reproduction in any medium or format, as long as you give appropriate credit to the original author(s) and the source, provide a link to the Creative Commons licence, and indicate if changes were made. The images or other third party material in this article are included in the article's Creative Commons licence, unless indicated otherwise in a credit line to the material. If material is not included in the article's Creative Commons licence and your intended use is not permitted by statutory regulation or exceeds the permitted use, you will need to obtain permission directly from the copyright holder. To view a copy of this licence, visit http://creativecommons.org/licenses/by/4.0/.

\section{Appendix A: Emission Measure Maps over the CH Lifetime}

Emission measure (Equation 3) integrated over different temperature bins, illustrating the difference in emission between $\mathrm{CH}$ and QS regions for the solar rotations not shown in Figure 6. (See Figures 11, 12, 13, 14, 15, 16, 17, 18, 19.) 

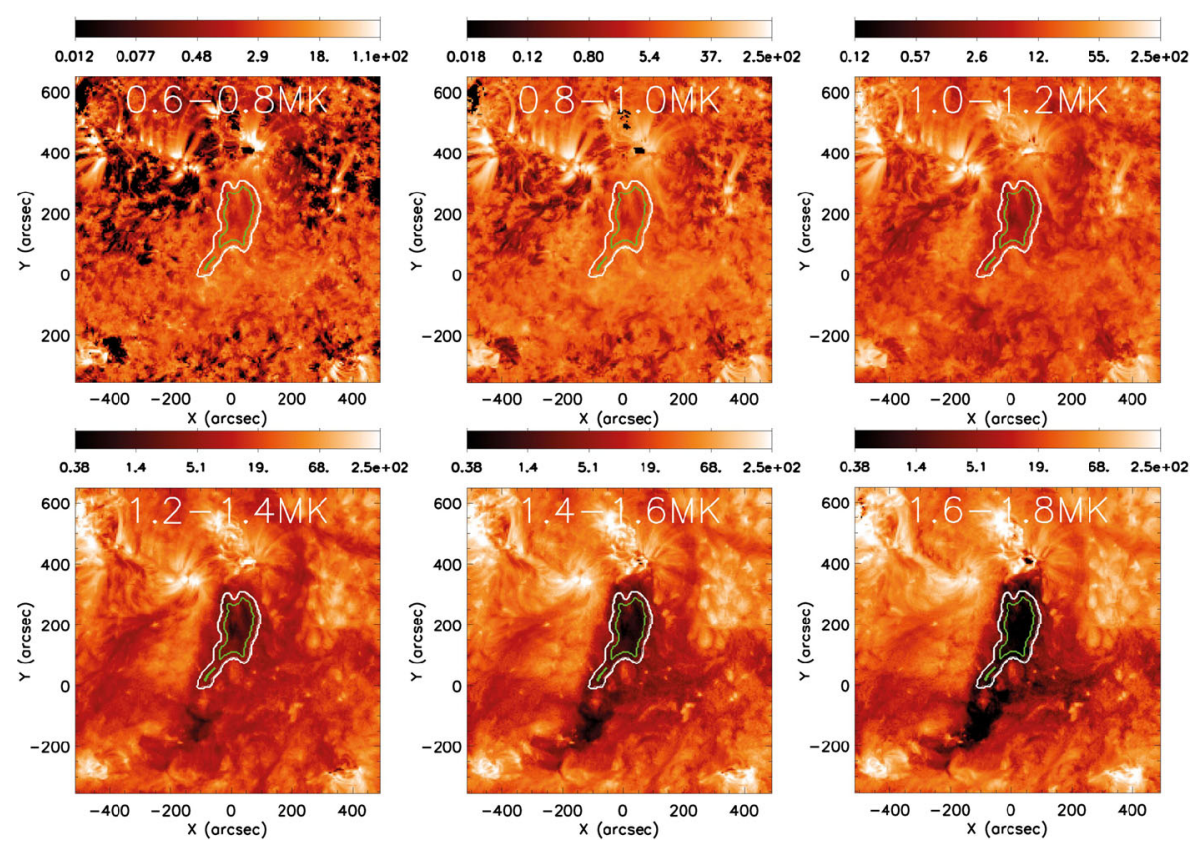

Figure 11 DEM integrated over six different temperature bins in the range 0.6 to $1.8 \mathrm{MK}$ in steps of $0.2 \mathrm{MK}$, yielding the total emission measure (EM) in units $\mathrm{cm}^{-5}$ at those temperature ranges. White contours indicate the initial, green the reduced $\mathrm{CH}$ boundaries. Observation from 15 February 2012.
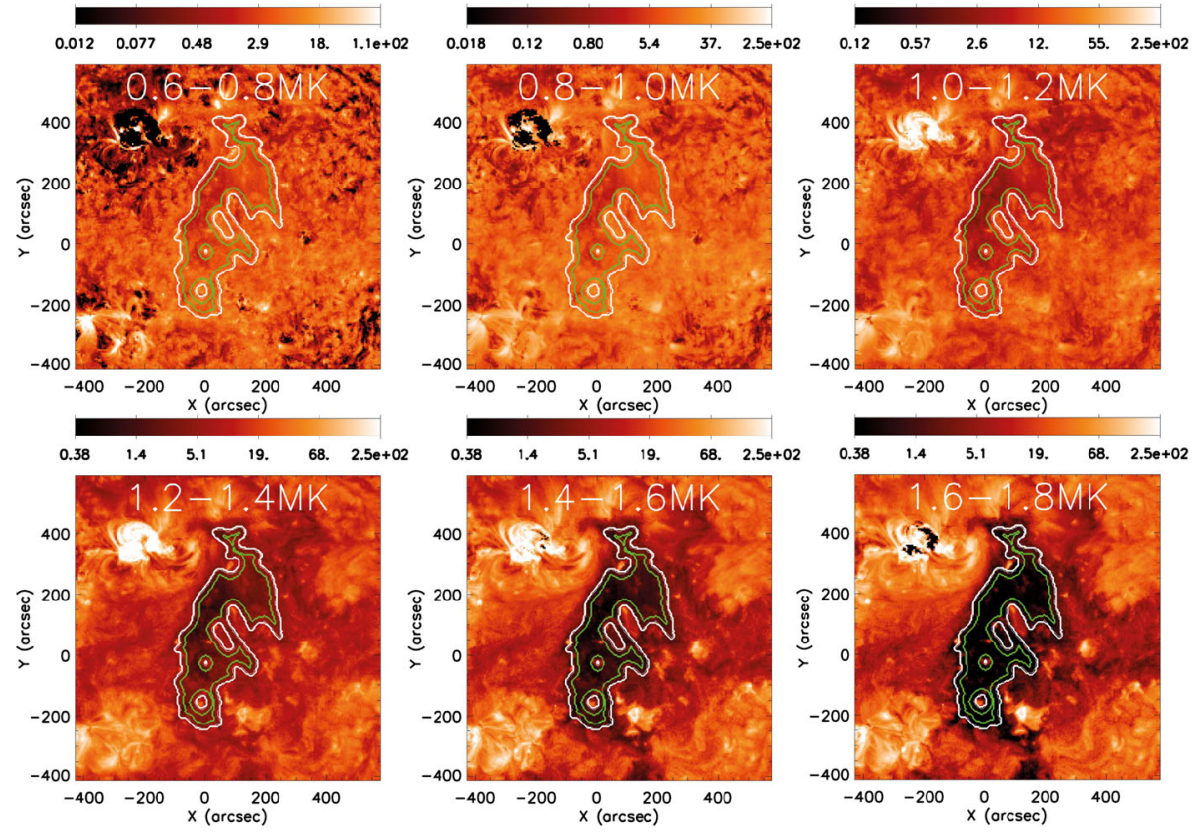

Figure 12 DEM integrated over six different temperature bins in the range 0.6 to $1.8 \mathrm{MK}$ in steps of $0.2 \mathrm{MK}$, yielding the total emission measure (EM) in units $\mathrm{cm}^{-5}$ at those temperature ranges. White contours indicate the initial, green the reduced $\mathrm{CH}$ boundaries. Observation from 14 March 2012. 

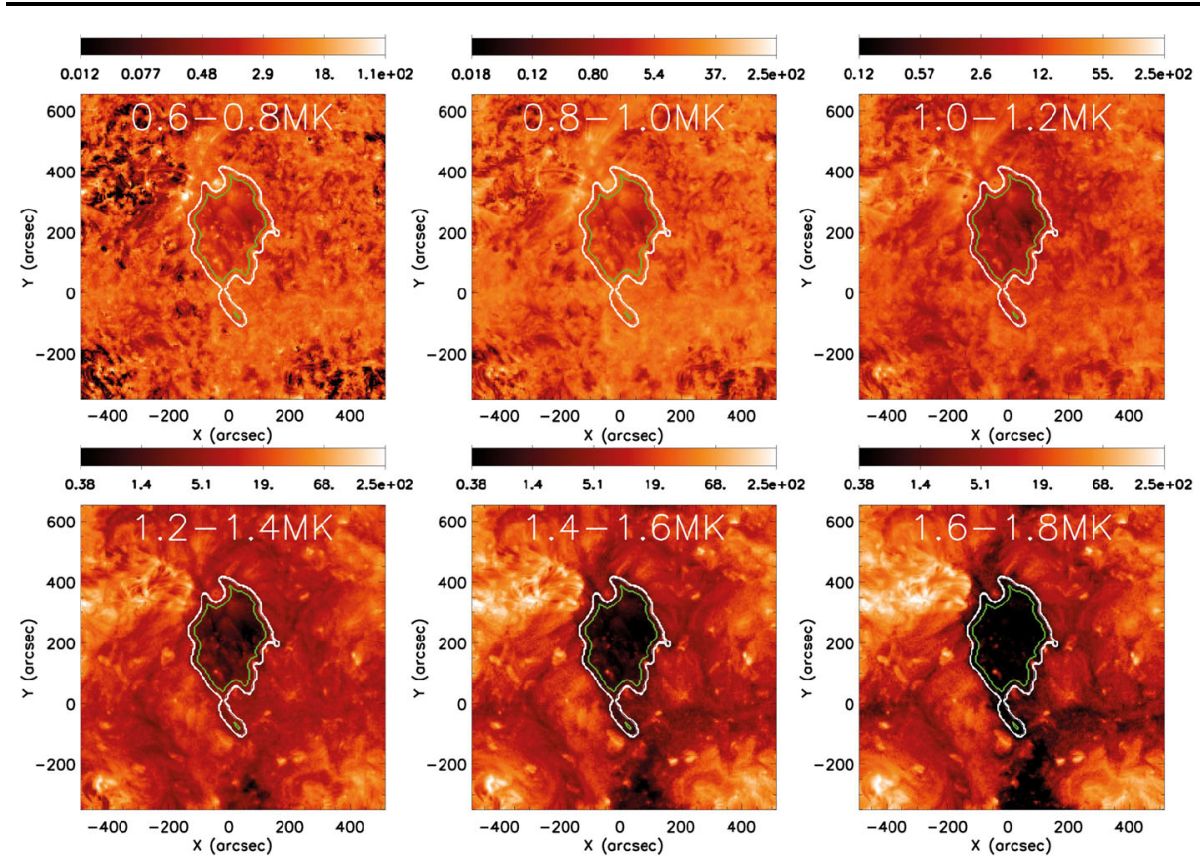

Figure 13 DEM integrated over six different temperature bins in the range 0.6 to $1.8 \mathrm{MK}$ in steps of $0.2 \mathrm{MK}$, yielding the total emission measure (EM) in units $\mathrm{cm}^{-5}$ at those temperature ranges. White contours indicate the initial, green the reduced $\mathrm{CH}$ boundaries. Observation from 09 April 2012.
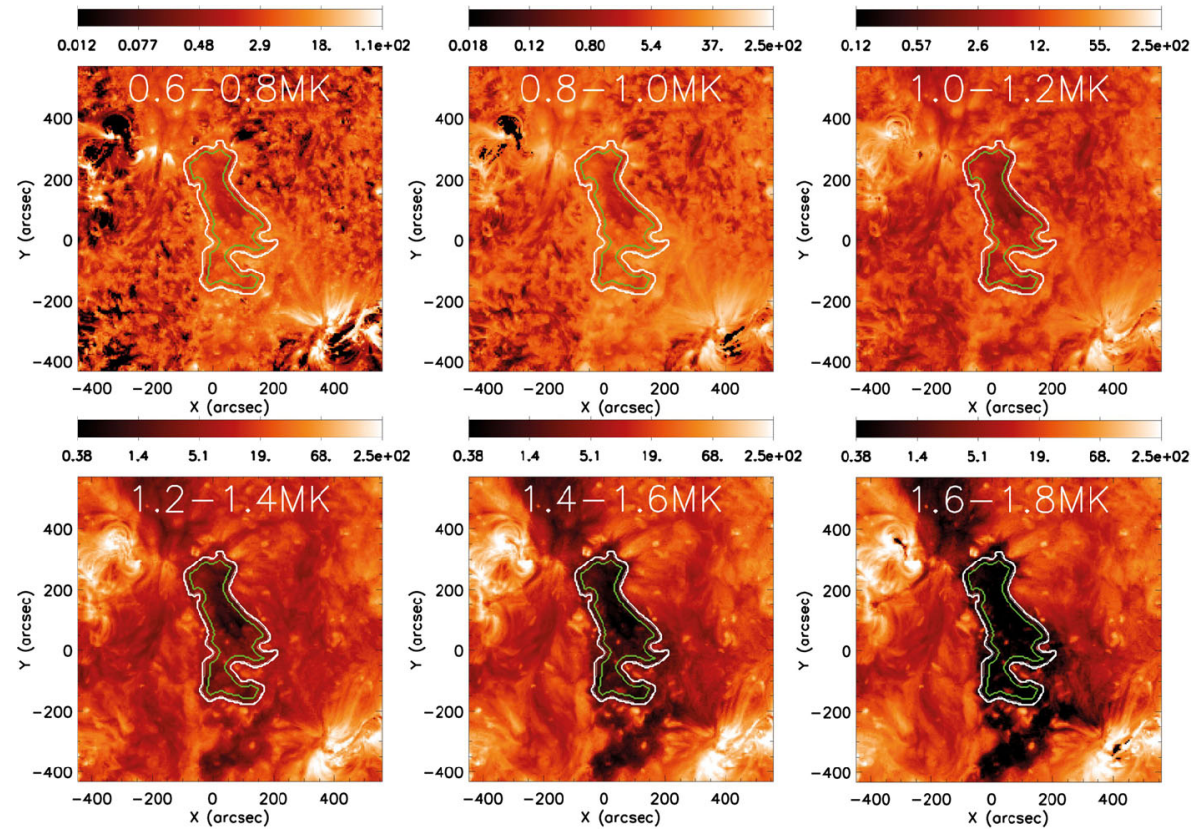

Figure 14 DEM integrated over six different temperature bins in the range 0.6 to $1.8 \mathrm{MK}$ in steps of $0.2 \mathrm{MK}$, yielding the total emission measure (EM) in units $\mathrm{cm}^{-5}$ at those temperature ranges. White contours indicate the initial, green the reduced $\mathrm{CH}$ boundaries. Observation from 06 May 2012. 

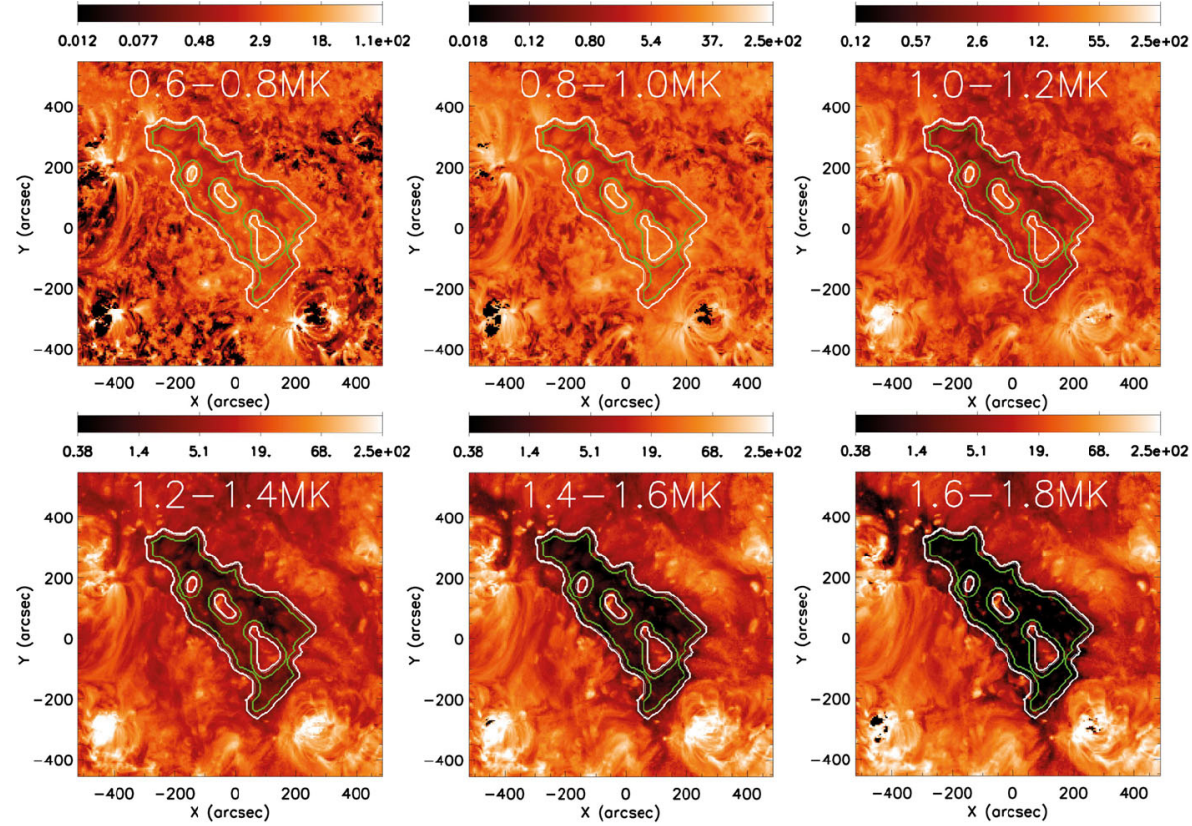

Figure 15 DEM integrated over six different temperature bins in the range 0.6 to $1.8 \mathrm{MK}$ in steps of $0.2 \mathrm{MK}$, yielding the total emission measure (EM) in units $\mathrm{cm}^{-5}$ at those temperature ranges. White contours indicate the initial, green the reduced $\mathrm{CH}$ boundaries. Observation from 30 June 2012.
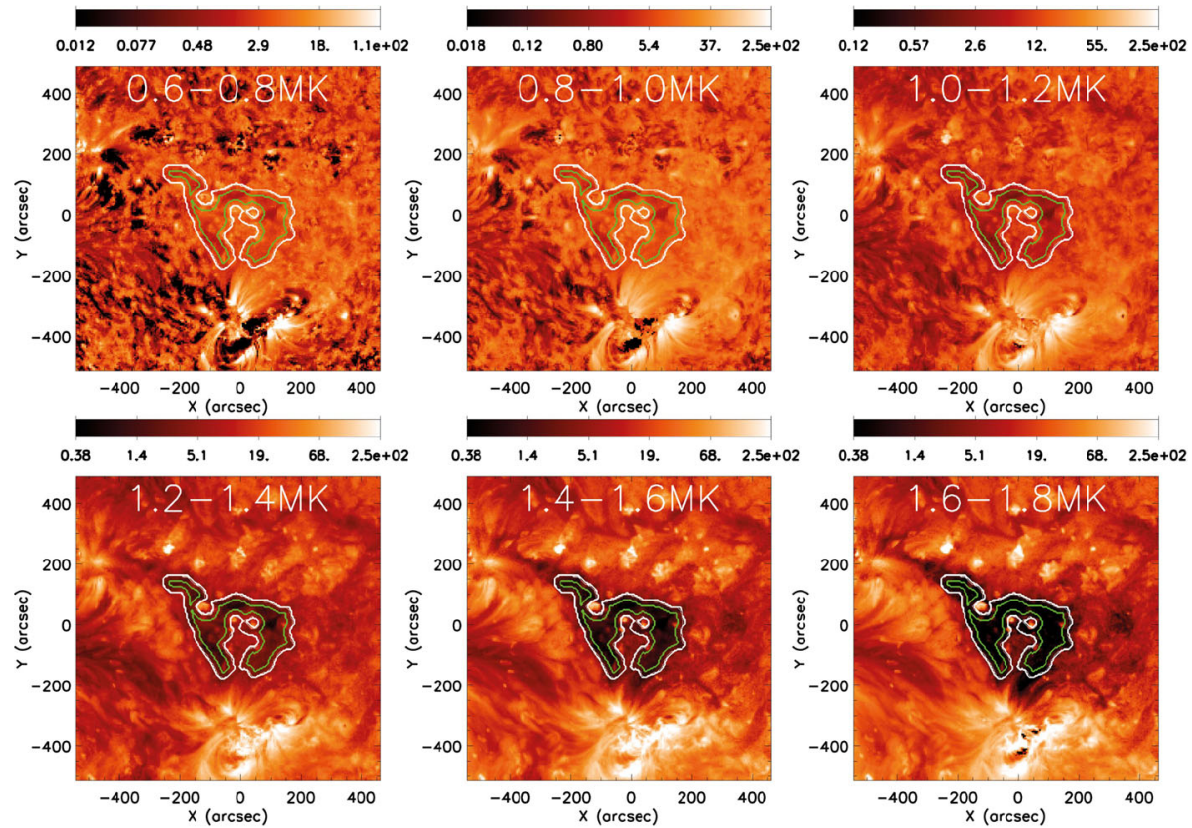

Figure 16 DEM integrated over six different temperature bins in the range 0.6 to $1.8 \mathrm{MK}$ in steps of $0.2 \mathrm{MK}$, yielding the total emission measure (EM) in units $\mathrm{cm}^{-5}$ at those temperature ranges. White contours indicate the initial, green the reduced CH boundaries. Observation from 26 July 2012. 


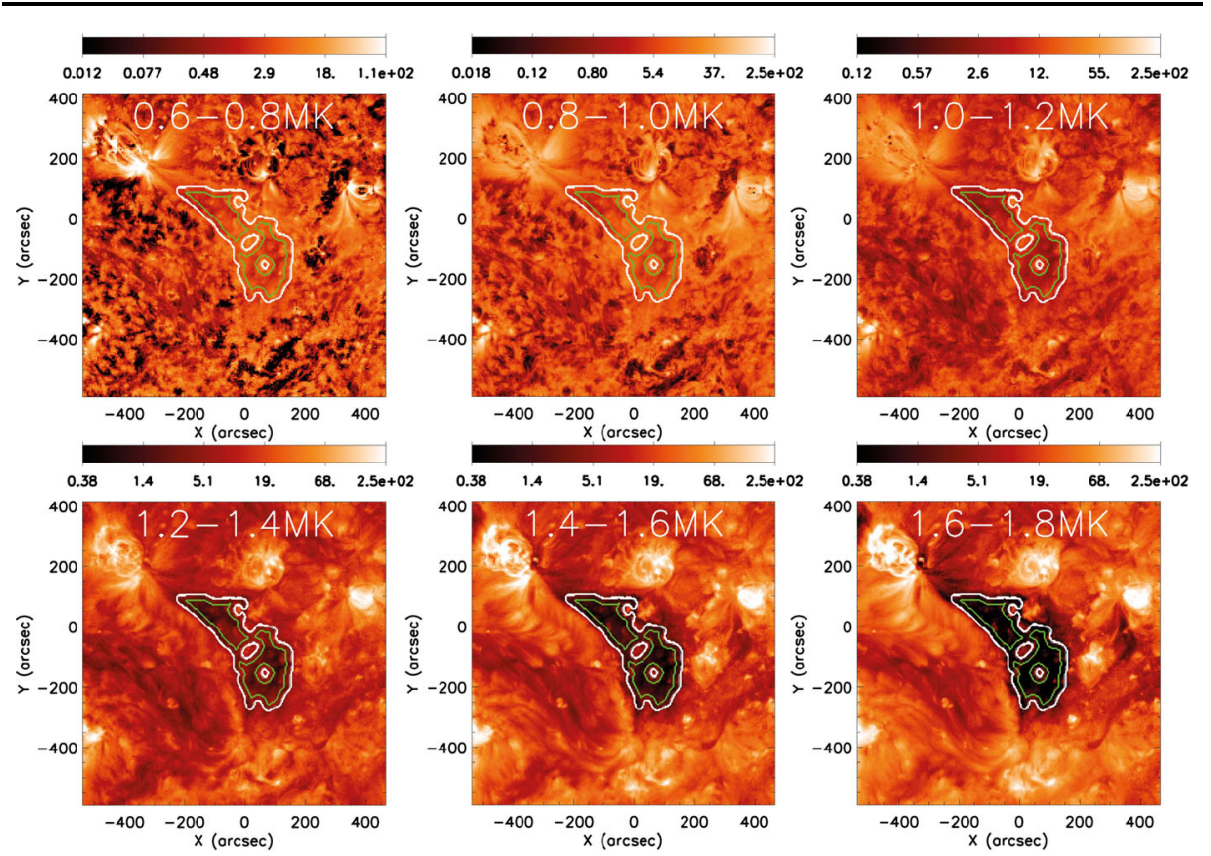

Figure 17 DEM integrated over six different temperature bins in the range 0.6 to $1.8 \mathrm{MK}$ in steps of $0.2 \mathrm{MK}$, yielding the total emission measure (EM) in units $\mathrm{cm}^{-5}$ at those temperature ranges. White contours indicate the initial, green the reduced CH boundaries. Observation from 22 August 2012.
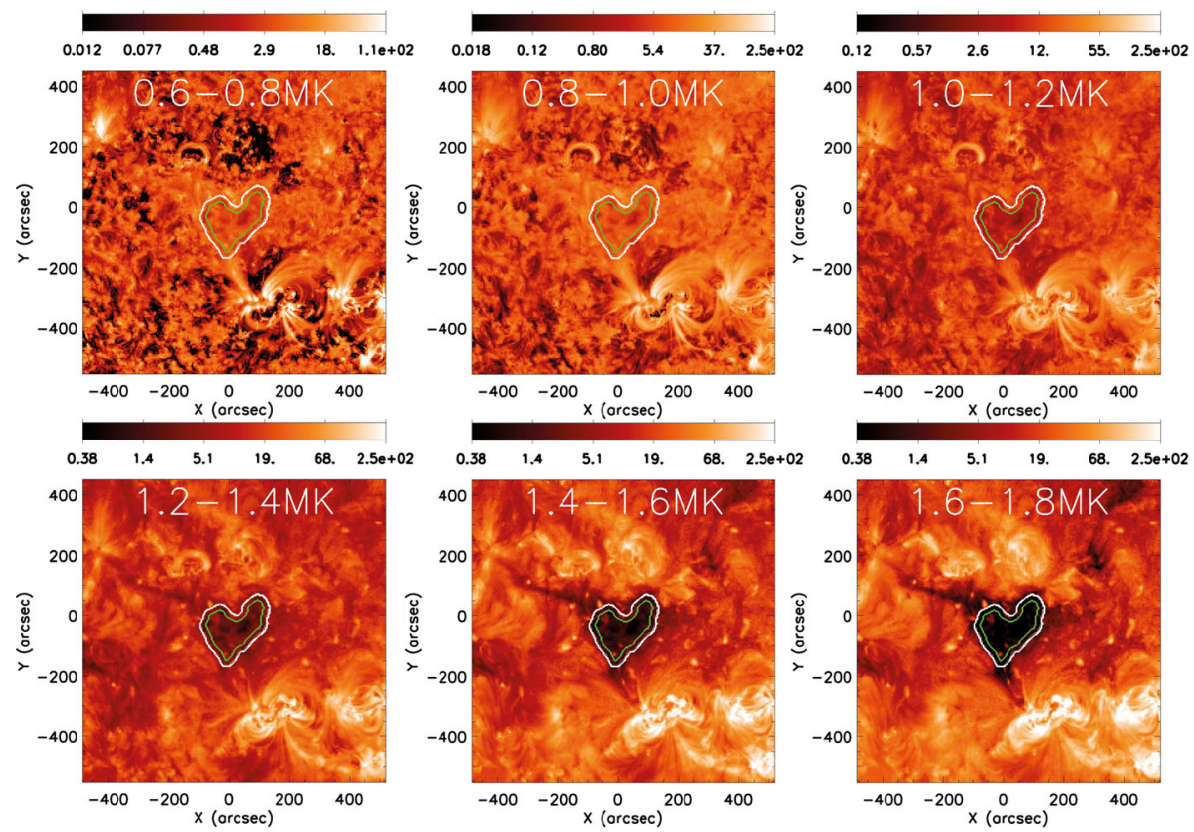

Figure 18 DEM integrated over six different temperature bins in the range 0.6 to $1.8 \mathrm{MK}$ in steps of $0.2 \mathrm{MK}$, yielding the total emission measure (EM) in units $\mathrm{cm}^{-5}$ at those temperature ranges. White contours indicate the initial, green the reduced CH boundaries. Observation from 18 September 2012. 

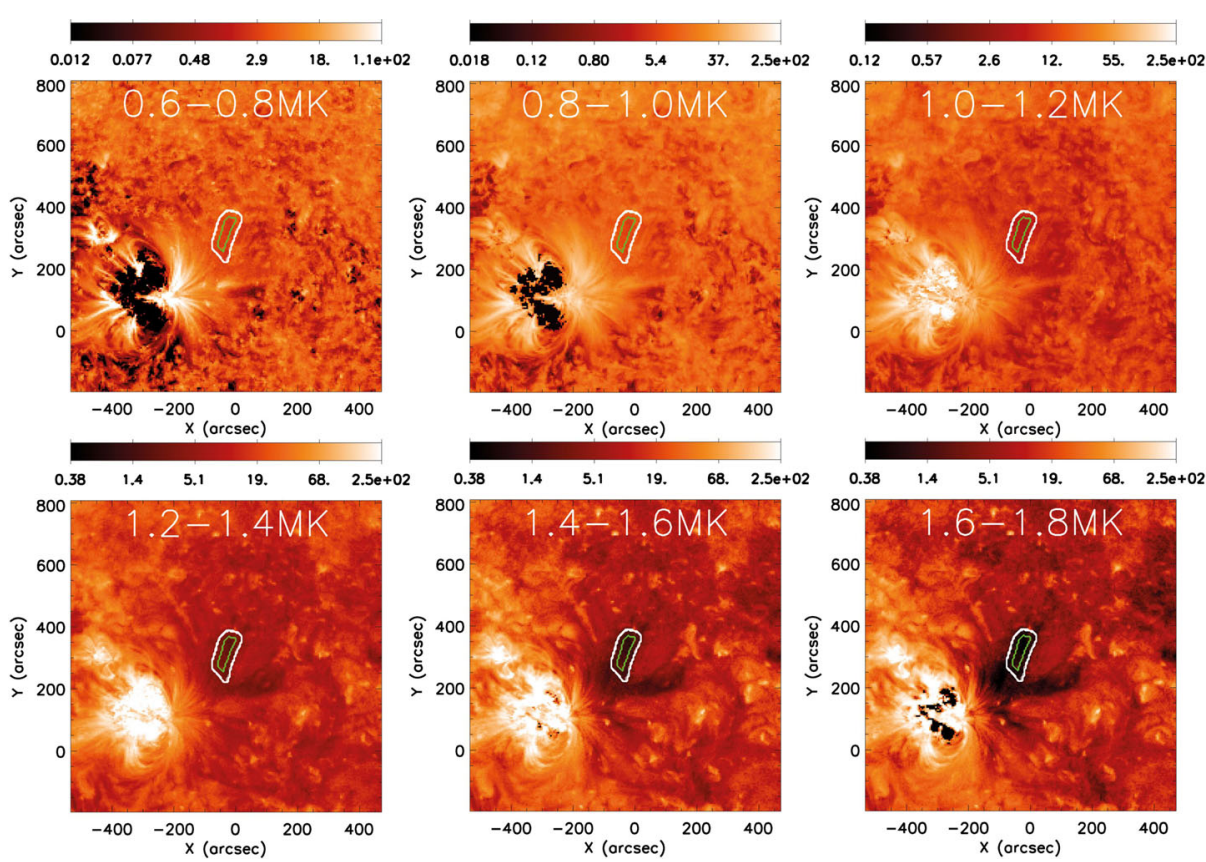

Figure 19 DEM integrated over six different temperature bins in the range 0.6 to $1.8 \mathrm{MK}$ in steps of $0.2 \mathrm{MK}$, yielding the total emission measure (EM) in units $\mathrm{cm}^{-5}$ at those temperature ranges. White contours indicate the initial, green the reduced $\mathrm{CH}$ boundaries. Observation from 14 October 2012.

\section{References}

Abramenko, V.I., Pevtsov, A.A., Romano, P.: 2006, Coronal heating and photospheric turbulence parameters: observational aspects. Astrophys. J. Lett. 646, L81. DOI. ADS.

Altschuler, M.D., Trotter, D.E., Orrall, F.Q.: 1972, Coronal holes. Solar Phys. 26, 354. DOI. ADS.

Cheng, X., Zhang, J., Saar, S.H., Ding, M.D.: 2012, Differential emission measure analysis of multiple structural components of coronal mass ejections in the inner corona. Astrophys. J. 761, 62. DOI. ADS.

Dere, K.P., Landi, E., Mason, H.E., Monsignori Fossi, B.C., Young, P.R.: 1997, CHIANTI - an atomic database for emission lines. Astron. Astrophys. Suppl. 125, 149. DOI. ADS.

Dere, K.P., Del Zanna, G., Young, P.R., Landi, E., Sutherland, R.S.: 2019, CHIANTI - an atomic database for emission lines. XV. Version 9, improvements for the X-ray satellite lines. Astrophys. J. Suppl. Ser. 241, 22. DOI. ADS.

Feldman, U.: 1998, FIP effect in the solar upper atmosphere: spectroscopic results. Space Sci. Rev. 85, 227. DOI. ADS.

Feldman, U., Widing, K.G.: 2003, Elemental abundances in the solar upper atmosphere derived by spectroscopic means. Space Sci. Rev. 107, 665. DOI. ADS.

Hahn, M., Landi, E., Savin, D.W.: 2011, Differential emission measure analysis of a polar coronal hole during the solar minimum in 2007. Astrophys. J. 736, 101. DOI. ADS.

Hannah, I.G., Kontar, E.P.: 2012, Differential emission measures from the regularized inversion of Hinode and SDO data. Astron. Astrophys. 539, A146. DOI. ADS.

Hassler, D.M., Dammasch, I.E., Lemaire, P., Brekke, P., Curdt, W., Mason, H.E., Vial, J.-C., Wilhelm, K.: 1999, Solar wind outflow and the chromospheric magnetic network. Science 283, 810. DOI. ADS.

Heinemann, S.G., Temmer, M., Hofmeister, S.J., Veronig, A.M., Vennerstrøm, S.: 2018a, Three-phase evolution of a coronal hole. I. $360^{\circ}$ remote sensing and in situ observations. Astrophys. J. 861, 151. DOI. ADS.

Heinemann, S.G., Hofmeister, S.J., Veronig, A.M., Temmer, M.: 2018b, Three-phase evolution of a coronal hole. II. The magnetic field. Astrophys. J. 863, 29. DOI. ADS. 
Heinemann, S.G., Temmer, M., Heinemann, N., Dissauer, K., Samara, E., Jerčić, V., Hofmeister, S.J., Veronig, A.M.: 2019, Statistical analysis and catalog of non-polar coronal holes covering the SDO-era using CATCH. Solar Phys. 294, 144. DOI. ADS.

Hofmeister, S.J., Veronig, A., Reiss, M.A., Temmer, M., Vennerstrom, S., Vršnak, B., Heber, B.: 2017, Characteristics of low-latitude coronal holes near the maximum of solar cycle 24. Astrophys. J. 835, 268. DOI. ADS.

Hofmeister, S.J., Utz, D., Heinemann, S.G., Veronig, A., Temmer, M.: 2019, Photospheric magnetic structure of coronal holes. Astron. Astrophys. 629, A22. DOI. ADS.

Hundhausen, A.J.: 1972, Coronal Expansion and Solar Wind, Physics and Chemistry in Space 5, Springer, Heidelberg. DOI. ADS.

Klimchuk, J.A.: 2006, On solving the coronal heating problem. Solar Phys. 234, 41. DOI. ADS.

Krieger, A.S., Timothy, A.F., Roelof, E.C.: 1973, A coronal hole and its identification as the source of a high velocity solar wind stream. Solar Phys. 29, 505. DOI.

Landi, E.: 2008, The off-disk thermal structure of a polar coronal hole. Astrophys. J. 685, 1270. DOI. ADS.

Landi, E., Feldman, U.: 2008, The thermal structure of an active region observed outside the solar disk. Astrophys. J. 672, 674. DOI. ADS.

Lemen, J.R., Title, A.M., Akin, D.J., Boerner, P.F., Chou, C., Drake, J.F., Duncan, D.W., Edwards, C.G., Friedlaender, F.M., Heyman, G.F., Hurlburt, N.E., Katz, N.L., Kushner, G.D., Levay, M., Lindgren, R.W., Mathur, D.P., McFeaters, E.L., Mitchell, S., Rehse, R.A., Schrijver, C.J., Springer, L.A., Stern, R.A., Tarbell, T.D., Wuelser, J.-P., Wolfson, C.J., Yanari, C., Bookbinder, J.A., Cheimets, P.N., Caldwell, D., Deluca, E.E., Gates, R., Golub, L., Park, S., Podgorski, W.A., Bush, R.I., Scherrer, P.H., Gummin, M.A., Smith, P., Auker, G., Jerram, P., Pool, P., Soufli, R., Windt, D.L., Beardsley, S., Clapp, M., Lang, J., Waltham, N.: 2012, The Atmospheric Imaging Assembly (AIA) on the Solar Dynamics Observatory (SDO). Solar Phys. 275, 17. DOI. ADS.

Pesnell, W.D., Thompson, B.J., Chamberlin, P.C.: 2012, The Solar Dynamics Observatory (SDO). Solar Phys. 275, 3. DOI. ADS.

Rotter, T., Veronig, A.M., Temmer, M., Vršnak, B.: 2012, Relation between coronal hole areas on the sun and the solar wind parameters at 1 AU. Solar Phys. 281, 793. DOI.

Tu, C.-Y., Zhou, C., Marsch, E., Xia, L.-D., Zhao, L., Wang, J.-X., Wilhelm, K.: 2005, Solar wind origin in coronal funnels. Science 308, 519. DOI.

Vanninathan, K., Veronig, A.M., Dissauer, K., Madjarska, M.S., Hannah, I.G., Kontar, E.P.: 2015, Coronal response to an EUV wave from DEM analysis. Astrophys. J. 812, 173. DOI. ADS.

Vršnak, B., Temmer, M., Veronig, A.M.: 2007, Coronal holes and solar wind high-speed streams: I. Forecasting the solar wind parameters. Solar Phys. 240, 315. DOI. ADS.

Wang, Y.-M.: 2010, On the relative constancy of the solar wind mass flux at 1 AU. Astrophys. J. Lett. 715, L121. DOI. ADS.

Wendeln, C., Landi, E.: 2018, EUV emission and scattered light diagnostics of equatorial coronal holes as seen by hinode/EIS. Astrophys. J. 856, 28. DOI. ADS.

Wilcox, J.M.: 1968, The interplanetary magnetic field. Solar origin and terrestrial effects. Space Sci. Rev. 8, 258. DOI. ADS. 